\title{
Use of pulmonary function test demographic data to identify high-risk patients for lung cancer screening
}

\author{
Amanda R. Jimenez'1, Arielle Schaeffer Weiss², Anthony C. Campagna3 \\ 1 Department of Medicine, Division of Pulmonary and Critical Care Medicine, Duke University \\ Health System, Durham, NC \\ 2 Department of Obstetrics and Gynecology, University of Washington \\ 3 Division of Pulmonary and Critical Care Medicine, Lahey Hospital and Medical Center, Burlington, MA, USA
}

\begin{abstract}
Lung Cancer is the primary cause of cancer-related death in the United States. We have recognized a need for novel methods of identification and consideration of enrollment into a lung cancer screening program for those at the highest risk of lung cancer.

Our primary goal was to determine if pulmonary function test (PFT) demographic data would be useful in identifying patients for lung cancer screening. We retrospectively reviewed PFTs performed at
\end{abstract}

Corresponding author: Amanda R. Jimenez, Medical Instructor, Department of Medicine, Division of Pulmonary and Critical Care Medicine, Duke University Health System, 3643 N. Roxboro St., Durham, NC 27704, USA. Tel.+001.585.7383034. E-mail: Amanda.Jimenez@duke.edu

Key words: Lung cancer screening; pulmonary function test; prevention.

Contributions: ARJ, conception and design, PFT / Lung Cancer Screening database creator, analysis and interpretation of data, drafting of paper and final approval of version to be published; ASW, analysis and interpretation of data, chart review, final approval of version to be published; ACC, drafting and revision of article for critically important intellectual content, subject expert opinion, final approval of version to be published.

Conflict of interest: The authors declare no conflict of interest.

Acknowledgements: The authors thank all members of the Lahey Hospital and Medical Center's "Rescue Lung Rescue Life" Lung Cancer Screening Committee for their collaboration on this project at many steps. Our great appreciation is extended to Brady McKee, MD, for review of this manuscript. This project also would not have been possible without the Lahey Hospital and Medical Center Burlington Campus PFT Lab, many thanks to them for their work obtaining accurate demographic data.

Received for publication: 22 October 2017

Accepted for publication: 28 Janaury 2018

(C) Copyright A.R. Jimenez et al., 2018

Tipografia PI-ME Editrice, Italy

Monaldi Archives for Chest Disease 2018; 88:891

doi: 10.4081/monaldi.2018.891

This article is distributed under the terms of the Creative Commons Attribution Noncommercial License (by-nc 4.0) which permits any noncommercial use, distribution, and reproduction in any medium. provided the original author(s) and source are credited.
Lahey Hospital \& Medical Center (LHMC) January 2012 through January 2013 and found that of patients identified as high risk of lung cancer, $89 \%$ had passed through our PFT lab but had not yet been screened. Investigation into the barriers of lung cancer screening to better understand how to increase appropriate enrollment then followed.

A total of 3098 PFTs were reviewed from January 2012 through January 2013 and 634 patients (20\%) were identified as high risk for lung cancer. Of 634 patients, 70 (11\%) were already in the LHMC lung cancer screening program. The remaining 564 patients (89\%) were not enrolled, and of these, it was found that 292 patients identified as high risk for lung cancer represented missed opportunities for screening. The remaining 272 patients were appropriately not screened with the three most common reasons being prior imaging with positive finding, lung cancer within five years, and provider discussed but scan not yet performed.

Appropriate enrollment in a lung cancer screening program may be increased with the careful use of demographic data obtained from a PFT lab.

\section{Introduction}

Lung cancer is the primary cause of cancer-related death in the United States [1]. The five-year survival rate has not changed over many decades as lung cancers are typically diagnosed at a later stage when curative procedures are not an option [1]. Numerous organizations now recommend low-dose CT (LDCT) scanning in patients determined to be high risk for developing lung cancer. Following the National Lung Cancer Screening Trial (NLST) results in 2011 reporting a 20-percent reduction in lung cancer specific mortality [2], Lahey Hospital and Medical Center (LHMC) started a Lung Cancer Screening Program in January of 2012. The LHMC Lung Cancer Screening Program is a multidisciplinary model to ensure appropriate, responsible screening and follow-up. Included in this model is a standardized CT lung imaging reporting and data system, or LungRADS, which reduces the $26.6 \%$ false-positive rate of screening observed in the NLST to $10 \%$ or less without sacrificing the overall sensitivity of detection of malignancy [3]. Combined with the results of NLST, Lahey's published reports on their initial and subsequent screening experiences provide additional evidence that early stage lung cancer may be diagnosed via an appropriately structured lung cancer screening program [4].

The NLST defined patients at high risk for lung cancer as having a 30 pack-year smoking history, 55-74 years of age, and actively smoking or having quit within the past 15 years [2]. As the definition of a highrisk group may change over time, effective methods to enroll these pa- 
tients are necessary. We have recognized this need to offer novel methods of identification and consideration of enrollment into a lung cancer screening program for those at the highest risk for lung cancer. We suspected that physicians from all departments at Lahey were under utilizing LDCT scans for lung cancer screening.

The following report describes the experience at LHMC with identification and recruitment of high risk patients for lung cancer screening through the pulmonary function test (PFT) lab. This report is the first published data on integration of the pulmonary function test lab into the lung cancer screening arena.

\section{Materials and Methods}

Our primary goal was to determine if PFT demographic data would be a useful tool in identifying patients for lung cancer screening, ultimately, leading to early detection of disease. At Lahey, PFT demographic data is displayed on the heading of each patient's PFT report and includes the patient's age, smoking status and pack-year history. We retrospectively reviewed PFTs performed at the LHMC Burlington campus between January 2012 and January 2013, a total of 13 months. This retrospective review was performed with approval from LHMC Institutional Review Board1.

Only patients meeting the NLST or National Comprehensive Cancer Network (NCCN) group 1 definition of high risk for lung cancer were included in this study - age 55 to 74 years, 30 pack years of smoking or greater, and active smoker or quit smoking within the past 15 years [5]. The United States Preventative Task Force (USPTF) recommendations for lung cancer screening were published after the first year of our data collection period and extended the age range for screening to 80 years [6]. The start date of our study preceded the USPTF definition, thus, our study used the NLST and NCCN group 1 age range of 55 to 74 years.

We previously reported that $89 \%$ of high-risk patients for lung cancer identified through our PFT lab at Lahey had not yet been screened. To maximize enrollment of these high-risk patients, prior to the use of our current electronic medical record, paper memos were sent to each ordering primary care physician (PCP), or to the provider who ordered the PFT, requesting they consider referring their patient for lung cancer screening. Responses of the PCPs, and other providers, to our request were then collected and analyzed.

\section{Results}

A total of 3098 PFTs were reviewed between January 2012 and January 2013. Of these, 634 patients (20\%) were identified as high-risk for lung cancer meeting the NCCN group 1 criteria. Of the 634 high risk patients, only 70 patients (11\%) were already in the Lahey lung cancer screening program, and the remaining 564 patients (89\%) were not enrolled. Characterization of our population is reported in Table 1. There were no significant differences between patients who were screened and those not screened.

PCP and other provider responses to our memo, suggesting consideration of lung cancer screening for their patient, were variable. Responses ranged from very positive with appreciation for our recruitment efforts to marginally negative. Some PCPs were concerned about adding to the volume of their already burdensome responsibility for follow-up on numerous imaging and laboratory results. There were also instances where the PCP disagreed with our suggestion based on information known to them that appropriately excluded their patient from screening. These reasons were not extractable from our PFT demographic data review. Due to this variable response, continued active recruitment with paper memos was aborted. Additional extensive chart review was performed on the 564 unscreened high risk patients to offer clarity of the information influencing PCP opinion. These reasons for not screening are reported in Table 2.

We determined that 292 patients (51.8\%) out of a total of 564 patients identified as high risk for lung cancer were missed opportunities for screening. These unscreened patients did not fall into any category in Table 2 . The remaining 272 patients (48.2\%) were appropriately not screened with the most common reasons bolded in Table 2 . These include the patient with prior imaging showing a positive finding already being followed, lung cancer within five years, or provider discussed but scan not yet performed.

\section{Discussion}

There are various ways in which high risk patients for lung cancer may be identified that reach beyond provider recognition. Implementing programs that utilize processes that are already occurring, such as pulmonary function testing, may increase screening numbers.

Our original hypothesis was that the PFT lab would be an ideal place to capture patients at high risk for lung cancer given the high likelihood of patients with a heavy smoking history referred for testing. The above results showing that $89 \%$ of high risk patients were unscreened suggested that the PFT lab may be an appropriate starting place to explore increasing lung cancer screening recruitment.

One reason for our low lung cancer screening enrollment of only $11 \%$ of high risk patients seen through the PFT lab may be because the time of this study was in the first year of the LHMC lung cancer screening program. If we were to repeat this study presently, we might expect a higher number of PFT patients to be screened due to ongoing PCP and provider education about lung cancer screening at Lahey and through other continuing medical education venues. Also, our current elec-

Table 1. Characteristics of patients referred for PFT's and at high risk for lung cancer during the study interval.

\begin{tabular}{lccc} 
Variable & Screened, $\mathbf{n}=70$ & Not screened, $\mathbf{n}=564$ & p value \\
Age & $62.9 \pm 6.0$ & $64.1 \pm 5.5$ & 0.0880 \\
\hline Pack years & $58.7 \pm 60.8$ & $51.3 \pm 26.3$ & 0.3238 \\
\hline Male & $39(55.7 \%)$ & $304(53.9 \%)$ & 0.7740 \\
\hline Active smoker & $32(45.7 \%)$ & $248(44.0 \%)$ & 0.7818 \\
\hline
\end{tabular}

${ }^{1}$ Lahey Clinic, Inc. IRB, Burlington, MA. Reference \# DR13-1312; initial approval date 3/8/13, amendment approval date 5/11/15. 
Table 2. Identification of reasons why high risk patients for lung cancer were not screened.

\begin{tabular}{|c|c|c|}
\hline Exclusion criteria / reasons why provider did not screen with LDCT & ned & patients, $\mathrm{n}=564$ \\
\hline Patient with known metastatic disease & 8 & $(1.4 \%)$ \\
\hline Patient with lung cancer within 5 years & 66 & $(11.7 \%)$ \\
\hline Patient with new symptoms concerning for lung cancer (hemoptysis, unexplained weight loss with smoking history) & 1 & $(0.2 \%)$ \\
\hline $\begin{array}{l}\text { Patient with symptoms concerning for active infection/inflammation and provider specifically states } \\
\text { this is why they will wait to order screening CT }\end{array}$ & 0 & $(0 \%)$ \\
\hline $\begin{array}{l}\text { Patient with prior imaging with positive pulmonary findings for which diagnostic follow-up } \\
\text { evaluation is incomplete }\end{array}$ & 122 & $(21.6 \%)$ \\
\hline Patient with inability to hold breath for 6 seconds & 0 & $(0 \%)$ \\
\hline Provider states the patient is unwilling for serial follow-up or has significantly limited life expectancy for other reason & 0 & $(0 \%)$ \\
\hline Scan was scheduled, the patient cancelled scan thereafter & 14 & $(2.5 \%)$ \\
\hline Evidence that a low-dose CT was ordered for screening purposes but not enrolled in the LHMC screening program & 6 & $(1.1 \%)$ \\
\hline $\begin{array}{l}\text { Provider discussed screening CT however planned outside of study timeframe or patient } \\
\text { lost to follow-up after screening scan ordered }\end{array}$ & 48 & $(8.5 \%)$ \\
\hline $\begin{array}{l}\text { Patient with prior imaging with positive pulmonary findings for which diagnostic follow-up evaluation } \\
\text { is incomplete + patient specifically states unwilling for serial follow-up, in addition }\end{array}$ & 1 & $(0.2 \%)$ \\
\hline $\begin{array}{l}\text { Patient with prior imaging with positive pulmonary findings for which diagnostic follow-up evaluation } \\
\text { is incomplete + low-dose CT ordered for screening purposes in future but not currently in program }\end{array}$ & 2 & $(0.4 \%)$ \\
\hline $\begin{array}{l}\text { Patient with prior imaging with positive pulmonary findings for which diagnostic follow-up evaluation is incomplete } \\
+ \text { subsequently evidence of loss of follow-up }\end{array}$ & 4 & $(0.7 \%)$ \\
\hline \#Appro & riatel & ned, $n=272$ \\
\hline No exclusion criteria met & 292 & $(51.8 \%)$ \\
\hline+2 & reeni & Inities, $n=292$ \\
\hline
\end{tabular}

tronic medical record now prompts physicians and other providers to consider lung cancer screening for patients who meet minimal criteria. Anecdotally, we have noticed primary care providers expressing less apprehension about following up on abnormal findings of a screening CT as the screening program provides follow-up support to these providers through a lung cancer screening program navigator and an active Medical Thoracic Oncology Conference (MTOC) which reviews these positive findings weekly. This may contribute to less resistance for discussion of screening with patients and thus increasing the referral for screening in those most appropriate.

Although this is the case at Lahey, all PFT labs may not report the demographic data required to assess for the appropriateness of lung cancer screening and as this study shows, PFT demographic data does not contain the factors listed in Table 2 which appropriately exclude screening. Exclusion criteria such as prior lung cancer or ongoing serial testing for a positive finding on chest $\mathrm{CT}$ are factors that may be addressed at a successful MTOC program. As with other organ system screening, providers are limited in what can be done about a patient who refuses screening or who is lost to follow-up. An additional barrier to screening that we have identified at Lahey is insurance coverage of scans. This may be less of an issue now that Medicare is covering screening in appropriate populations up to age 77 [7].

In conclusion, the results from this study show that use of the PFT lab demographic data can be a useful tool to increase lung cancer screening enrollment in conjunction with appropriate clinical input from primary care providers.

\section{References}

1. American Cancer Society. Cancer Facts \& Figures 2016. Atlanta: American Cancer Society; 2016. https:/www.cancer.org/research/ cancer-facts-statistics/all-cancer-facts-figures/cancer-facts-figures2016.html (date last accessed, 7/20/17).

2. The National Lung Screening Trial Research Team. Reduced lungcancer mortality with low-dose computed tomographic screening. N Engl J Med 2011;365:395-409.

3. McKee BJ, Regis SM, McKee AB, Flacke S, Wald C. Performance of ACR Lung-RADS in a Clinical CT Lung Screening Program. J Am Coll Radiol 2015;12:273-276.

4. McKee BJ, McKee AB, Flacke S, Lamb C, Hesketh PJ, Wald C. Initial experience with a free high-volume, low-dose CT lung cancer screening program. J Am Coll Radiol 2013;10:586-592.

5. National Comprehensive Cancer Network. NCCN Guidelines For Lung Cancer Screening (V.1.2012). http//www.nccn.org/professionals/ physician_gls/pdf/lung_screening.pdf (date last accessed, 7/20/17).

6. U.S. Preventive Services Task Force. Final recommendation statement: Lung cancer: Screening, December 2016. Available at: https://www.uspreventiveservicestaskforce.org/Page/Document/ RecommendationStatementFinal/lung-cancer-screening (date last accessed, 7/20/17).

7. Medicare. Lung Cancer Screening. Accessed January 1, 2017. Available at: https://www.medicare.gov/coverage/lung-cancerscreening.html (date last accessed, 7/20/17). 